\section{The Effects of Hand Grip Strength and Clinical Findings to Quality of Life in Fibromy- algia Syndrome}

\author{
Halil Harman ${ }^{1 *}$, Kadriye Öneş ${ }^{2}$ and Ata Bora Ayna ${ }^{3}$ \\ ${ }^{1}$ Department of Physical Medicine and Rehabilitation, Rheumatology, \\ Sakarya University, Sakarya, Turkey \\ ${ }^{2}$ Istanbul Physical Therapy-Rehabilitation Education and Research and \\ Hospital, Istanbul, Turkey
}

${ }^{3}$ Department of Physical Medicine and Rehabilitation, Rheumatology, Uludağ University, Bursa, Turkey

\begin{abstract}
Background: Fibromyalgia Syndrome (FMS) can be defined as a chronic musculoskeletal disease with widespread pain and tender points on specific anatomical regions. FMS affects quality of life and causes severe chronic pain, and is associated to high prevalence of emotional disturbances including depression.

Objective: The aim of study was to investigate the possible relationship between clinical findings, hand grip strength and quality of life in patients with FMS.

Material and methods: Fifty consecutive women who fulfilled the 1990 American college of Rheumatology criterial for FMS were included in this study and compared with 40 age and BMI matched female healthy controls. Pain assessment was performed Visual Pain Scales (VPS) and Functional Pain Scale (FPS). Saehan hydraulic hand grip dynamometer was used for assessing patient and control groups hand grip strength. Beck depression inventory was used for evaluating patient and control groups' mental status. For patients' functional assessment were used fibromyalgia impact scale. Short Form 36 (SF-36) were investigated for quality of life of patients and the control group. It has a high reliability and validity for FMS patients.

Results: In FMS patients, the mean number of tender points was $15.0 \pm 1.79$ (range, 12-18), the mean VPS score was $7.70 \pm 1.75$ $\mathrm{cm}$, the mean FPS score was $6.64 \pm 1.42 \mathrm{~cm}$. The most common application complaints were body pain, back pain and headache. In our study, particularly morning stiffness, nonrestorative sleep, paresthesia complaints were found statistically significant effects on quality of life and depressive mood $(p<0.05)$. In patients with FMS,
\end{abstract}

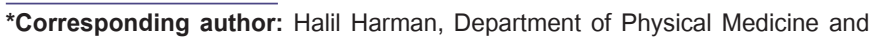
Rehabilitation, Rheumatology, Sakarya University, Sakarya, Turkey, Tel: +90 02642552106; Fax: +90 02642552105; E-mail: drhharman@yahoo.com

Citation: Harman H, Öneş K, Ayna AB (2015) The Effects of Hand Grip Strength and Clinical Findings to Quality of Life in Fibromyalgia Syndrome. J Orthop Res Physiother 1: 019.

Received: October 28, 2015; Accepted: December 01, 2015; Published: December 15, 2015 hand grip strength was inversely correlated with SF 36 total scores and SF-36 subgroup scores $(p<0.05)$.

Conclusion: Decreasing hand grip strength was influenced by neither the intensity of pain nor the motivation of patients. Muscle weakness in FMS patients can be responsible for physical inactivity due to the pain and physical decondition of muscles. We suggested that hand grip strength can be used for as a marker of quality of life not for diagnostic evaluation.

Keywords: Fibromyalgia; Hand grip strength; Quality of life

\section{Introduction}

Fibromyalgia Syndrome (FMS) can be defined as a chronic musculoskeletal disease with widespread pain and tender points on specific anatomical regions. In most patients, sleep disturbance, fatigue, stiffness, numbness may be accompanied with disease $[1,2]$.

In recent studies indicated the role of central mechanisms on FMS pathogenesis and this lead to new expansions on FMS treatment [3]. FMS patients have less physical and psychological condition compared with normal population [4]. Decreasing on functional muscle strength and endurance have shown in age-and sex-controlled studies $[5,6]$. In this study we aimed to investigate the possible relationship between clinical findings, hand grip strength and quality of life in patients with FMS.

\section{Material and Methods}

Fifty women who fulfilled the 1990 American college of Rheumatology criterial for FMS were included in this study and compared with 40 age and BMI matched female healthy controls between 2009 April and 2011 April. The study patients were randomly selected from outpatient clinic of physical medicine and rehabilitation and control group was selected health professionals. All patients and controls are between the age of 20-50 years old. All FMS patients were newly diagnosed. Patients and controls had no history of current therapy (pharmacologic or physical training); and had no history of any systemic or rheumatic disease, malignant or infective disease, cervical disc herniation, radiculopathy, myelopathy, myofascial pain syndrome, brain surgery, shoulder surgery, carpal tunnel surgery or trauma.

Tender points were examined using the protocol described by Wolfe et al., [7]. A score for the number of tender points was obtained ranging from 11 to 18 . All patients had pain bilaterally in the neck-shoulder region and in the lumbosacral-hip region, with radiation of pain to the extremities. In all cases, the pain was perceived as more severe in one-half of the body.

Pain assessment was performed Visual Pain Scales (VPS) and Functional Pain Scale (FPS). In VPS assessment, no pain is numbered 0 , the most severe pain is numbered 10 point, moderate pain is numbered 5 point. In addition to pain assessment, fatigue, morning stiffness are asked to patients. In FPS, lack of pain is numbered 0 point, 4 points is showed tolerable but prevents some activities, 8 points is showed that could not tolerate pain and passive movements are blocked.

The approach features use of a 0-3 scale, in which 0 corresponds to no morning stiffness, 1 to 30 minutes, 2 to 1 hour, and 3 to up to 
1 hour for evaluating morning stiffness. The presence of fatigue (1) or the absence thereof (0) was scored.

In our study FMS patients also be evaluated in terms of subgroups, and were classified into 4 groups like Muller's [8] study. According to this classification, group 1 has only increased sensitivity to pain, group 2 has depression affected by chronic pain, group 3 primary depression with chronic pain, group 4 FMS patients consists of somatization disorder.

Saehan hydraulic hand grip dynamometer was used for assessing patient and control groups hand grip strength. The dominant hand was tested. The test was performed in a sitting position, with the upper arm parallel to the trunk, the elbow at 90-degree flexion, and the forearm and hand in the zero position. The test was repeated 3 times. The time between each test was 1 minute and the best results were expressed as kilograms of force.

Beck Depression Inventory (BDI) was used for evaluating patient and control groups' mental status. According to this scale, $0-12$ points indicate minimal depression, 13-18 point mild depression, 19-28 point moderate depression, 29-63 point severe depression [9].

For patients' functional assessment were used Fibromyalgia Impact Scale (FIQ). This scale consists of 10 items and the validity and reliability for Turkey was conducted by Sarmer and colleagues in 2000 [10].

Short Form 36 (SF-36) was investigated for quality of life of patients and the control group. Mental, physical and total scores of SF 36 were calculated. It has a high reliability and validity for FMS patients [11].

\section{Statistical Analysis}

We used SPSS for Windows 16.0 program in our study. As descriptive statistics mean, standard deviation and frequencies were determined. Upon univariate analysis, the Mann-Whitney U test was used to compare continuous variables and the chi-squared test to compare categorical variables. Spearman's correlation coefficients between clinical and hand grip strength were calculated. A one-way ANOVA was used to test for differences among the four group means. A P value less than 0.05 was considered to indicate statistical significance.

\section{Results}

The study is included 50 patients diagnosed with FMS and 40 healthy controls. There were no significant differences between groups concerning age, height, weight and Body Mass Index (BMI) $(\mathrm{p}>0.05)$.

In FMS patients, the mean number of tender points was $15.0 \pm$ 1.79 (range, 12-18), the mean VPS score was $7.70 \pm 1.75 \mathrm{~cm}$, the mean FPS score was $6.64 \pm 1.42 \mathrm{~cm}$. In healthy controls, the mean number of tender points was $5.0 \pm 0.79$ (range, $1-11$ ), the mean VPS score was $2.50 \pm 0.24 \mathrm{~cm}$, the mean FPS score was $3.24 \pm 0.55 \mathrm{~cm}$. There were significant difference between healthy control and patient group concerning these values $(\mathrm{p}<0.001)$. The most common application complaints of FMS patients are widespread body pain (Figure 1). The painless symptoms in FMS patients are shown in figure 2.

In FMS, outside of pain, there were many symptoms that affect quality of life and mood. In our study, particularly morning stiffness, nonrestorative sleep, paresthesia complaints were found statistically significant correlation with BDI scores (respectively, $\mathrm{r}=0.451$,

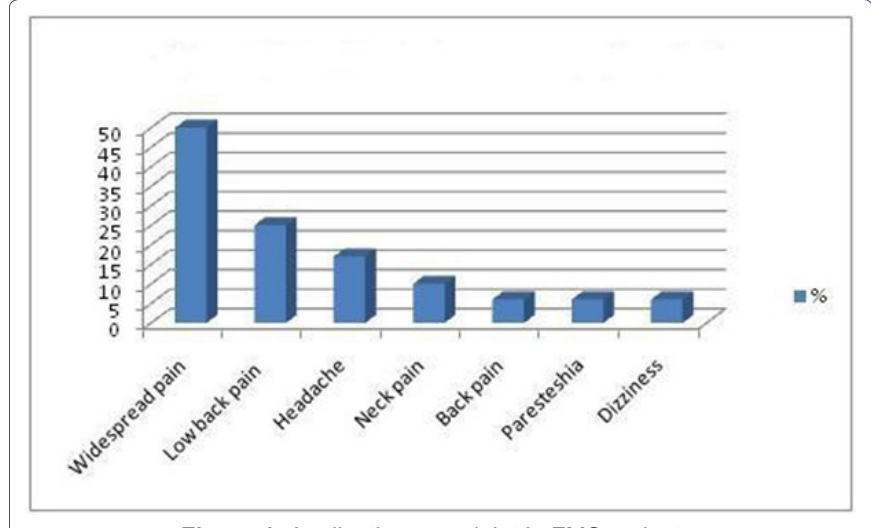

Figure 1: Application complaint in FMS patients.

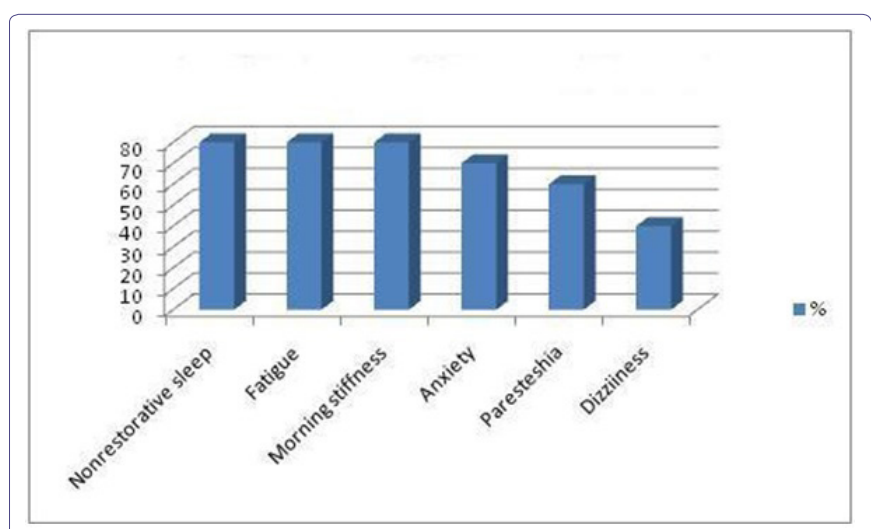

Figure 2: Painless symptoms in FMS patients.

$\mathrm{p}=0.01 ; \mathrm{r}=0.354, \mathrm{p}=0.05 ; \mathrm{r}=0.412, \mathrm{p}=0.024)$. Therefore, paresthesia and morning stiffness were positively correlated with SF 36 total scores (respectively, $r=0.412, p=0.022, r=0.457$, $\mathrm{p}=0.001)$.

Nonrestorative sleep and morning stiffness were lower in non-depressive patients (Group 1 and Group 4) than depressive patients (Group 2 and 3) ( $\mathrm{p}=0.001$ ).

$\% 36$ of FMS patients were classified like group 1. Group 2, group 3 and group 4 were classified $\% 30, \% 28$ and \% 6, respectively. The mean values of patient group's hand grip strength was $25.76 \pm 5.52$ $\mathrm{kg} /$ force and the mean values of control group's hand grip strength $33.95 \pm 5.66 \mathrm{~kg} /$ force. Hand grip strength was lower in FMS patients than in controls. These values were statistically significant $(\mathrm{p}<0.001)$. In patients with FMS, hand grip strength was inversely correlated with SF 36 total scores $(r=0.432, p=0.011)$. FIQ total scores did not correlate with hand grip strength $(\mathrm{r}=-0.145, \mathrm{p}=0.3 .14)$ (Figure 3 ). Other parameters was not correlated hand grip strength $(\mathrm{p}>0.05)$. In healthy controls, there was no correlation between hand grip strength and SF 36 total scores $(r=0.114, p=0.123)$. SF 36 total, physical health and mental health scores were shown in table 1.

BDI and VPS scores were significantly higher in FMS groups compared with healthy controls $(\mathrm{p}<0.05)$. SF-36 total, physical health and mental health scores were statistically significantly differences between FMS subgroups depression by the Muller's [8] study ( $\mathrm{p}<0.001$, $\mathrm{p}<0.001, \mathrm{p}<0.001$, respectively). In the depression group, total SF 36 scores were significantly higher (Table 1 ).

\section{Discussion}

FMS is the most common rheumatologic disease after osteoarthritis [3]. There is not an effective treatment strategy for all 


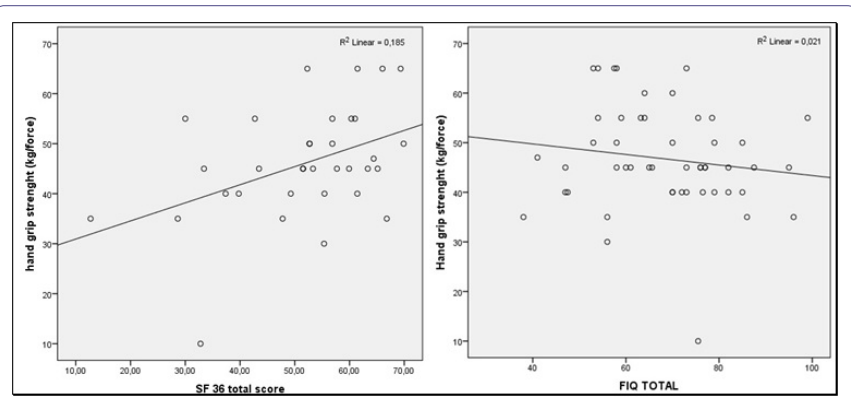

Figure 3: Relationship between hand grip strength and SF 36 total scores, FIQ total scores. isokinetic muscle strength in patients with FMS [14]. In present study, muscle strength was evaluated with isometric measurement. FMS patients were likely to reduce isometric muscular strength compared with control group. In a similar study, Maquet et al., had found statistically significant difference between patient group and control group for hand grip strength $(20 \pm 9 \mathrm{~kg} /$ force, $27 \pm 7 \mathrm{~kg} /$ force, $\mathrm{p}<0.01)$. In this study, isokinetic muscle strength were measured in FMS group and \% 56 lower results were obtained [15]. Sahin et al., had found decreased hand grip strength in FMS group and the results were correlated with pulmonary muscles strength [16]. Mannerkorpi et al., had evaluated physical performance in FMS and healthy controls with physical performance tests. As a result, patients with FMS had reduced at physical performance capabilities [17].

\begin{tabular}{|c|c|c|c|c|c|}
\hline & $\begin{array}{c}\text { Group } 1 \\
\text { (No psychiatric condition) }\end{array}$ & $\begin{array}{c}\text { Group 2 } \\
\text { (pain related depression) }\end{array}$ & $\begin{array}{c}\text { Group 3 } \\
\text { (Depression with } \\
\text { concomittant FMS) }\end{array}$ & $\begin{array}{c}\text { Group } 4 \\
\text { (somatization) }\end{array}$ & p value across categories \\
\hline Hand grip strength (kg/force) & $47.89 \pm 10.31$ & $47.66 \pm 6.77$ & $47.5 \pm 8.42$ & $41.67 \pm 13.86$ & 0.078 \\
\hline FIQ total scores & $69.90 \pm 13.30$ & $73.66 \pm 12.85$ & $73.17 \pm 15.21$ & $75.21 \pm 5.23$ & 0.094 \\
\hline BDI total scores & $13.21 \pm 7.90$ & $22.86 \pm 11.65$ & $28.28 \pm 15.21$ & $27.01 \pm 10.23$ & 0.006 \\
\hline SF 36 physical health & $57.83 \pm 7.20$ & $47.63 \pm 8.94$ & $43.26 \pm 8.54$ & $57.54 \pm 15.69$ & 0.034 \\
\hline SF 36 mental health & $63.07 \pm 6.14$ & $47.54 \pm 5.21$ & $42.82 \pm 6.41$ & $56.12 \pm 5.42$ & 0.001 \\
\hline SF 36 total scores & $61.41 \pm 4.81$ & $49.63 \pm 9.23$ & $44.05 \pm 5.68$ & $60.38 \pm 15.69$ & 0.002 \\
\hline
\end{tabular}

Table 1: Clinical evaluation and hand grip strength of FMS patients with subgroups.

patients because of uncertain etiopathogenesis. FMS affects quality of life and causes severe chronic pain, emotional disturbances including anxiety, depression.

According to 1990 ACR classification criteria, all FMS patients should have pain bilaterally in the neck-shoulder region and in the lumbosacral-hip region, with radiation of pain to the extremities. In addition, all the pain should be perceived as more severe in one-half of the body [4]. In 2002 Crofford and colleagues criticized to ACR classification crtiteria because of focusing the pain and ignoring fatigue, cognitive disorders, sleep disturbance and psychological disorders. They explained that neuroendocrine axis anomalies may be the cause of painless symptoms in patients with FMS [12]. Wilke et al., was noted low specificity (\% 81) in the ACR classification criteria [13]. In our study, nonrestorative sleep, fatigue, morning stiffness was the most common painless symptoms. We found that particularly nonrestorative sleep, morning stiffness and paresthetic complaint were correlated with decreased quality of life and depressed mood. Therefore, pain is important for the diagnosis of FMS but for assessing quality of life we should attach importance to painless symptoms. The cause of these symptoms is still unknown.

We investigated the subgroups of FMS patients accordingly to the study of Muller et al., in 2007 [8]. But we did not find a significant difference between subgroups and grip strength. Howewer, SF-36 total scores was higher in non-depressive group. At the same time nonrestorative sleep and morning stiffness was a less common presenting symptom in non-depressive patients. This also showed that nondepressive patients had better life quality compared with depressive patients. For appropriate treatment program of FMS patients, morning stiffness and sleep patterns should be questioned.

Evaluation of hand grip strength between FMS patients and normal healthy subjects was the main aim of this study. Measuring maximal voluntary isometric muscle contraction is the oldest method to assess muscle strength. Jacobsen et al., found that measurement of isometric muscle strength is equivalent with
All this studies indicated that hand grip strength is reduced in patients with FMS and this reduction is influenced by neither the intensity of pain nor the motivation of patients. Also many studies have shown that patients with FMS have impaired physical performance and mental health compared with other painful disease $[18,19]$. We have found significantly less physical functioning and mental health SF-36 scores in FMS. This reduction should be secondary to deterioration of physical condition or reduction to physical performance. Low maximum voluntary muscle strength in patients with FMS are not diagnostic. It can be seen together myofascial pain syndrome, rheumatoid arthritis and other rheumatic conditions.

Hand grip strength measurement should be used for assessment of quality of life with FMS rather than diagnosis crtiteria. Indeed, we did not find any correlation with tender points, FIQ scores and BDI scores with grip strength but SF36 total scores was significantly correlated with decreased grip strength.

FMS patients often have many disability in activities of daily living. Psychological factors may play an important role on this situation. In many studies was shown significant deterioriation in quality of life and mental health in patients with FMS [20,21]. This situation impacted on their social and business lives, and significant economic burden is due to loss of labor in addition health care costs [22]. Verbunt et al., suggested that disability of FMS patients was appeared in clinic like deteroriation of mental health. At the same time, patients with FMS were significantly higher rates of psychological stress compared with complex regional pain syndrome and chronic low back pain. Effect on the quality of life is very high compared to other diseases [19].

Hoffmann et al., were found significantly lower mental and physical SF-36 scores in FMS patients compared to other diseases [18]. We have achieved similar results with our study. FMS patients had lower mental health, physical health and general health SF-36 scores compared with control group. As noted other studies, FMS is characterized low mental health scores. 
Citation: Harman H, Önȩ̧ K, Ayna AB (2015) The Effects of Hand Grip Strength and Clinical Findings to Quality of Life in Fibromyalgia Syndrome. J Orthop Res Physiother 1: 019.

Being a cross-sectional study that consists of a small population size are some of the important limitations of our study. Still the results are worth discussing. Also, ACR 2010 criteria for FMS could be more valuable for this study. But we had designed this study between 2009 and 2011.

As a result, decreasing hand grip strength was influenced by neither the intensity of pain nor the motivation of patients. Consequently muscle weakness can be linked to peripheral and central mechanisms [23]. Several studies found no correlation in muscle histopathologically and muscle metabolism in FMS etiopathogenesis $[24,25]$. Muscle weakness in FMS patients can be responsible for blood flow changes in the central nervous system, physical inactivity due to the pain and physical decondition of muscles [26]. We suggested that hand grip strength can be used for as a marker of quality of life not for diagnostic evaluation.

\section{References}

1. Burkham J, Harris ED (2005) Fibromyalgia: A chronic pain syndrome. In: Harris ED, Budd RC, Firenstein GS, Genovese MC, Servent JS, et al. (eds.). Kelley's Rheumatology. Elsevier Saunders, Philadelphia, USA. Pg no: 522-536.

2. Gran JT (2003) The epidemiology of chronic generalized musculoskeletal pain. Best Pract Res Clin Rheumatol 17: 547-561.

3. Price DD, Staud R (2005) Neurobiology of fibromyalgia syndrome. J Rheumatol Suppl 75: 22-28.

4. Wortmann RL (1994) Searching for the cause of fibromyalgia: is there a defect in energy metabolism? Arthritis Rheum 37: 790-793.

5. Mannerkorpi K, Burckhardt CS, Bjelle A (1994) Physical performance characteristics of women with fibromyalgia. Arthritis Care Res 7: 123-129.

6. Valim V, Feldman D, Olivers L, Silva LL, Suda AL, et al. (1999) Low anaerobic threshold and maximum oxygen uptake in fibromyalgia. Arthritis \& Rheumatism 42: 477 .

7. Wolfe F, Smythe HA, Yunus MB, Bennet RM, Bombardier C, et al. (1990) The American College of Rheumatology 1990 criteria for classification of fibromyalgia. Report of the multicenter criteria committee. Arthritis Rheum 33: 160-172.

8. Müller W, Schneider EM, Stratz T (2007) The classification of fibromyalgia syndrome. Rheumatol Int 27: 1005-1010.

9. Kapci EG, Uslu R, Turkcapar H, Karaoglan A (2008) Beck Depression Inventory II: evaluation of the psychometric properties and cut-off points in a Turkish adult population. Depress Anxiety 25: 104-110.

10. Sarmer S, Ergin S, Yavuzer G (2000) The validity and reliability of the Turkish version of the Fibromyalgia Impact Questionnaire. Rheumatol Int 20: 9-12.
11. Strömbeck B, Ekdahl C, Manthorpe R, Wikström I, Jacobsson L (2000) Health related quality of life in primary Sjögren's syndrome, rheumatoid arthritis and fibromyalgia compared to normal population data using SF-36. Scand J Rheumatol 29: 20-28.

12. Crofford LJ, Clauw DJ (2002) Fibromyalgia: Where are we a decade after the American college of Rheumatology classification criteria were developed? Arthritis Rheum 46: 1136-1138.

13. Wilke WS (2009) New developments in the diagnosis of fibromyalgia syndrome: say goodbye to tender points? Cleve Clin J Med 76: 345-352.

14. Jacobsen S, Wildschiødtz G, Danneskiold-Samsøe B (1991) Isokinetic and isometric muscle strength combined with transcutaneous electrical muscle stimulation in primary fibromyalgia syndrome. J Rheumatol 18: 1390-1393.

15. Maquet D, Croisier JL, Renard C, Crielaard JM (2002) Muscle performance in patients with fibromyalgia. Joint Bone Spine 69: 293-299.

16. Sahin G, Ulubaș B, Calikoğlu M, Erdoğan C (2004) Handgrip strength, pulmonary function tests, and pulmonary muscle strength in fibromyalgia syndrome: is there any relationship? South Med J 97: 25-29.

17. Mannerkorpi K, Burckhardt CS, Bjelle A (1994) Physical performance characteristics of women with fibromyalgia. Arthritis Care Res 7: 123-129.

18. Hoffman DL, Dukes EM (2008) The health status burden of people with fibromyalgia: a review of studies that assessed health status with the SF-36 or the SF-12. Int J Clin Pract 62: 115-126.

19. Verbunt JA, Pernot DH, Smeets RJ (2008) Disability and quality of life in patients with fibromyalgia. Health Qual Life Outcomes 6: 8.

20. Jiao J, Vincent A, Cha SS, Luedtke CA, Oh TH (2015) Association of abuse history with symptom severity and quality of life in patients with fibromyalgia. Rheumatol Int 35: 547-553.

21. Tutoglu A, Boyaci A, Koca I, Celen E, Korkmaz N (2014) Quality of life, depression, and sexual dysfunction in spouses of female patients with fibromyalgia. Rheumatol Int 34: 1079-1084.

22. Jeffery DD, Bulathsinhala L, Kroc M, Dorris J (2014) Prevalence, health care utilization, and costs of fibromyalgia, irritable bowel, and chronic fatigue syndromes in the military health system, 2006-2010. Mil Med 179: 1021-1029.

23. Flodin P, Martinsen S, Löfgren M, Bileviciute-Ljungar I, Kosek E, et al. (2014) Fibromyalgia is associated with decreased connectivity between pain- and sensorimotor brain areas. Brain Connect 4: 587-594.

24. Klemp P, Nielsen HV, Korsgård J, Crone $P$ (1982) Blood flow in fibromyotic muscles. Scand J Rehabil Med 14: 81-82.

25. Yunus MB, Kalyan-Raman UP, Masi AT, Aldag JC (1989) Electron microscopic studies of muscle biopsy in primary fibromyalgia syndrome: a controlled and blinded study. J Rheumatol 16: 97-101.

26. Littlejohn $G$ (2015) Neurogenic neuroinflammation in fibromyalgia and complex regional pain syndrome. Nat Rev Rheumatol 11: 639-648. 